\section{Ovarialkarzinom: Betablockertherapie im Fokus}

\author{
Aus präklinischen Versuchen ist bekannt, dass die anhaltende Aktivierung \\ adrenerger Rezeptoren das Wachstum von Ovarialkarzinomen fördert. Unklar \\ war bisher, ob Patientinnen von Betablockern profitieren.
}

$\mathrm{n}$ vitro blockiert z. B. Propranolol die Synthese des VEGF („vascular endothelial growth factor") und verhindert damit die Tumor-Neoangiogenese. Außer dem Wachstum von Ovarialkarzinomen fördert die anhaltende Aktivierung v.a. $\beta_{2}$-adrenerger Rezeptoren auch die Metastasierung.

Um den Einfluss von $\beta_{1}$-selektiven im Vergleich zu nicht selektiven Betablockern auf den Parameter Gesamtüberleben (OS) aufzuhellen, analysierten USGynäkologen retrospektiv die Daten von 1.425 Patientinnen, die zwischen 2000 und 2010 wegen eines epithelialen Ovarialkarzinoms mindestens eine platinbasierte Chemotherapie erhalten hatten. 269 Patientinnen hatten eine Betablockade erhalten, 75 von ihnen mit einem nicht selektiven Präparat. Der Betablo- cker wurde v. a. wegen Hypertonie, aber auch wegen Arrhythmien und zur Versorgung nach einem Myokardinfarkt eingesetzt.

Patientinnen mit Betablocker-Therapie überlebten deutlich länger als Betroffene ohne (median 47,7 vs. 42 Monate; $\mathrm{p}=0,04)$. Unter der Therapie mit nicht selektiv wirksamen Präparaten war das OS mehr als doppelt so lang wie unter $\beta_{1}$-selektiven Substanzen (median 94,9 vs. 38 Monate; $\mathrm{p}<0,001$ ). Das bestätige die Bedeutung $\beta_{2}$-adrenerger Rezeptoren in der Genese des Ovarialkarzinoms sowie den Nutzen nicht selektiver Betablocker, so die Forscher.

Zwar hatten mit 75 insgesamt nur 5\% aller Patientinnen nicht selektive Betablocker erhalten. Dennoch stufen Kommentatoren das Ergebnis als „dramati- schen Überlebensvorteil“" ein, der weitere klinische Studien rechtfertige.

Als eine Erklärung für die Wirksamkeit von Betablockern beim Ovarialkarzinom führen die Forscher an, dass die Präparate das immunologische Microenvironment verbessern helfen. Eine Schlüsselrolle spiele in diesem Zusammenhang das unter anderem durch Stress induzierte Zytokin Interleukin 6, dessen Synthese durch die Betablockade gedrosselt wird und das u.a. an der Entstehung des Ovarialkarzinoms beteiligt ist.

Fazit: Bei Frauen mit Ovarialkarzinom ist die Therapie mit nicht selektiven Betablockern scheinbar mit einer deutlichen Verlängerung des OS assoziiert. In 2 Studien wird der Nutzen von Propranolol aktuell geprüft.

Peter Leiner

Watkins JL et al. Clinical impact of selective and nonselective beta-blockers on survival in patients with ovarian cancer. Cancer. 2015;121(19): 3444-51. Kommentar: Bunch KP, Annunziata CM Are beta-blockers on the therapeutic horizon for ovarian cancer treatment? Cancer. 2015;121(19):3380-3.

\section{Beginn der Chemotherapie nicht zu lange hinauszögern bei Ovarialkarzinomen}

\section{Forscher untersuchten, ob die Zeit zwischen Operation und Beginn der Chemotherapie einen Einfluss auf das Überleben von Patientinnen mit fortgeschrittenem Ovarialkarzinom hat.}

$\mathrm{D}$ as Intervall zwischen Operation und Beginn der Chemotherapie könnte für das Überleben von Frauen mit neu diagnostiziertem Ovarialkarzinom entscheidend sein. Eine Metaanalyse hatte ergeben, dass das Rezidivrisiko steigt und das Gesamtüberleben sinkt, wenn sich der Beginn der Chemotherapie nach der Operation verzögert. Dieser Zusammenhang wurde nun in einer nachträglich durchgeführten Ad-hoc-Analyse der Phase-III-Studie 218 der Gynecologic Oncology Group erneut untersucht.

1.718 Patientinnen mit neu diagnostiziertem, fortgeschrittenem Ovarialkarzinom in den Stadien III oder IV wurden in dieser Analyse ausgewertet, darunter solche mit vollständiger Resektion, einer
Resterkrankung mit geringem Volumen $(<1 \mathrm{~cm})$ oder suboptimaler Resektion $(>1 \mathrm{~cm})$. Bei Patientinnen im Stadium III, nicht aber im Stadium IV, musste als Einschlusskriterium eine makroskopische oder palpable Resterkrankung nach der Operation vorliegen. Eigentliches Ziel der Studie war es, den Nutzen von Bevacizumab zusätzlich zur Chemotherapie in der Induktions- und Erhaltungstherapie zu untersuchen. Die mediane Zeit zwischen Operation und Beginn der Chemotherapie betrug 31 Tage, bei $27 \%$ der Patienten war sie länger als 40 Tage.

In der multivariaten Analyse war die Zeit bis zum Beginn der Chemotherapie prädiktiv für das Gesamtüberleben ( $p<0,001)$, wobei die Gruppe mit voll- ständiger Resektion (Stadium IV) ein erhöhtes Sterberisiko hatte, wenn die Zeit bis zum Beginn der Chemotherapie 25 Tage überstieg. Beispielsweise stieg das Sterberisiko bei weißen Patienten mit kompletter Resektion nach 25 Tagen um $27 \%$, für jede Verlängerung des Intervalls um $10 \%$. Am stärksten war der Einfluss des Zeitintervalls auf das Überleben bei Frauen mit mikroskopischer Resterkrankung.

Fazit: Das Überleben von Frauen mit fortgeschrittenem Ovarialkarzinom im Stadium IV könnte negativ beeinflusst werden, wenn die Chemotherapie mehr als 25 Tage nach der Operation beginnt. In weiteren Studien sollten prospektiv mögliche Gründe untersucht werden, die das Intervall zwischen Operation und Beginn der Chemotherapie beeinflussen.

Judith Neumaier

Tewari KS et al. Early Initiation of Chemotherapy following Complete Resection of Advanced Ovarian Cancer Associated with Improved Survival: An NRG Oncology/Gynecologic Oncology Group Study. Ann Oncol. 2016 Jan;27(1):114-21. 